\title{
Effets de l'aménagement en courbe de niveau avec différentes doses de fertilisation sur les rendements du sorgho dans les localités de Kolokani et de Diéma au Mali
}

\author{
Moussa ZEROME ${ }^{1 *}$, Kalifa TRAORE ${ }^{2}$, Mahamoudou FAMANTA $^{3}$, \\ Boubacar Soumaïla MAIGA ${ }^{2}$, Oumar SAMAKE ${ }^{2}$ et Moise Anewin TOGO ${ }^{4}$

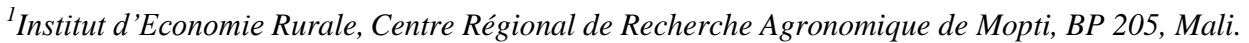 \\ ${ }^{2}$ Institut d'Economie Rurale, Centre Régional de Recherche Agronomique de Sotuba, Laboratoire Sol-Eau- \\ Plante BP 262, Bamako, Mali. \\ ${ }^{3}$ Institut Polytechnique de Formation et de Recherche Appliquée (IPR/IFRA) de Katibougou BP 06, Koulikoro \\ ${ }^{4}$ World Vision, Badalabougou (face au palais de la culture), Rue 56 Porte 403, BP 2347, Bamako, Mali. \\ *Auteur correspondant ; E-mail : zeromemoussa@yahoo.fr; Tel. (+223) 76044013 /65 309229.
}

\section{REMERCIEMENTS}

Nous adressons nos sincères remerciements à World Vision pour le financement de cette étude.

\section{RESUME}

Les objectifs de cette étude consistaient à caractériser les propriétés physico-chimiques des sols et évaluer l'effet de l'aménagement en courbe de niveau sur les rendements du sorgho. Des échantillons composites de sol ont été analysés pour connaître la fertilité initiale des parcelles d'expérimentation. Le dispositif expérimental était le split plot avec 20 répétitions. Les parcelles principales étaient constituées par les parcelles aménagées et parcelles non aménagées (NA) ; les parcelles secondaires par quatre niveaux de fertilisation. La dimension d'une parcelle était de $150 \mathrm{~m}^{2}$. La fumure organique (320 kg/ha) et le NPK 17-17-17 $(19,2 \mathrm{~kg} / \mathrm{ha})$ ont été apportés en micro-dose au semis. Le semis a été fait à l'écartement $80 \mathrm{~cm} \mathrm{x} 40 \mathrm{~cm}$. L'urée a été enfouie en micro-dose $(3,2 \mathrm{~kg} / \mathrm{ha})$ à $10 \mathrm{~cm}$ des plants de sorgho en début de montaison après une pluie. Les sols avaient une faible fertilité. L'aménagement en courbe de niveau (ACN) a produit $845 \mathrm{~kg} / \mathrm{ha}$ de rendement grain, $2084 \mathrm{~kg} / \mathrm{ha}$ de paille contre $227 \mathrm{~kg} / \mathrm{ha}$ et $499 \mathrm{~kg} / \mathrm{ha}$ pour le témoin. L'ACN a augmenté significativement $(\mathrm{p}<0,001)$ les rendements grain et paille de plus $175 \%$. L'ACN est reproductible partout au Sahel et dans d'autres régions à écologie similaire.

(C) 2019 International Formulae Group. All rights reserved

Mots clés : ACN, NA, micro-dose, rendement sorgho, Mali.

\section{Effects of contour ridge tillage with different rates of fertilization on sorghum yields in Kolokani and Diema areas, Mali}

\begin{abstract}
The objectives of this study were to characterize the physicochemical properties of soils and to evaluate effect of contour ridge tillage on sorghum yields. Composite soil samples were analyzed in order to know initial fertility of experimental plots. Experimental device was the split plot with 20 repetitions. Main plots consisted of contoured plots and no-contoured plots (NCRT); secondary plots by four levels of fertilization.
\end{abstract}


Size of a plot was $150 \mathrm{~m}^{2}$. Organic manure $\left(320 \mathrm{~kg} \mathrm{ha}^{-1}\right)$ and NPK 17-17-17 (19.2 $\left.\mathrm{kg} \mathrm{ha}^{-1}\right)$ were provided in micro-dose at sowing. Sowing was done at $80 \mathrm{~cm} \mathrm{x} 40 \mathrm{~cm}$ spacing. Urea was applied in micro-dose $(3.2 \mathrm{~kg}$ $\left.\mathrm{ha}^{-1}\right)$ at $10 \mathrm{~cm}$ from sorghum plants at beginning of elongation after a rain. Soils had low fertility. Contour ridge tillage (CRT) provided $845 \mathrm{~kg} \mathrm{ha}^{-1}$ of grain yield and $2084 \mathrm{~kg} \mathrm{ha}^{-1}$ of straw versus $227 \mathrm{~kg} \mathrm{ha}^{-1}$ and $499 \mathrm{~kg}$ $\mathrm{ha}^{-1}$ for control. CRT increased significantly $(\mathrm{p}<0.001)$ grain and straw yields by more than $175 \%$. CRT is replicable throughout Sahel and other areas of similar ecology.

(C) 2019 International Formulae Group. All rights reserved

Keywords: CRT, NCRT, micro-dose, sorghum yield, Mali.

\section{INTRODUCTION}

Le ruissellement est important dans les zones soudano-sahéliennes et les dégâts sont énormes. Bertrand et Gigou (2000) mentionnaient que dans les zones nord soudaniennes et celles soudano-sahéliennes, l'intensité sur 30 minutes pourrait atteindre 56 $\mathrm{mm} \mathrm{h}^{-1}$ en moyenne une fois chaque année et $78 \mathrm{~mm} \mathrm{~h}^{-1}$ tous les 10 ans. L'érosion hydrique est accentuée par les forts ruissellements. Le ruissellement est néfaste à la production agricole parce que, d'une part il réduit les disponibilités en eau pour les cultures et les arbres du parc arboré et, d'autre part, il peut entraîner l'appauvrissement du sol par décapage des horizons superficiels (Kizito et al., 2005).

Plusieurs technologies ont été mise au point pour lutter contre les pertes en eau par ruissellement dont les objectifs principaux étaient d'améliorer la productivité des cultures, en favorisant la conservation de l'eau du sol dans les champs cultivés (Gigou et al., 2006 ; Kablan et al., 2008; Traoré et al., 2017a ;Traoré et al., 2017b ; Birhanu et al., 2018). Des augmentations de rendement de $30 \%$ en moyenne sur les céréales ont été rapportées par l'utilisation de la technologie d'aménagement en courbes de niveau (Traoré et al., 2002; 2003). Par ailleurs, la technologie d'aménagement en courbes de niveau a considérablement augmenté les rendements de maiis de $24 \%$ et le C organique du sol de $26 \%$ en Gambie, de $12 \%$ de $\mathrm{C}$ et $30 \%$ sur la production des mil-sorgho à Siguidolo (Mali), et de $14 \%$ sur la production en systèmes arachidier de Nioro, Sénégal. Ces augmentations de $\mathrm{C}$ organique des sols et de rendement des cultures sont dues à trois facteurs à savoir la réduction de l'érosion du sol, la croissance accrue des cultures, résultant de la plus grande capture des précipitations et l'augmentation de la croissance et la densité des arbustes et arbres, résultant de l'eau du sous-sol qui a augmenté, ce qui entraîne la réduction du ruissellement (Doumbia et al., 2009).

En dépit de l'introduction de la technologie d'aménagement en courbe de niveau dans la zone Mali-sud dans les années 1990, leurs effets sur les rendements des cultures dans les autres zones agricoles du Mali en combinaison avec la micro-dose d'engrais ont été peu documentés. Les objectifs de la présente étude consistaient à caractériser les propriétés physico-chimiques des sols et à évaluer l'effet de l'aménagement en courbe de niveau sur les rendements du sorgho en zone sahélienne de Kolokani et de Diéma au Mali.

\section{MATERIEL ET METHODES}

\section{Localisation des sites d'étude}

L'étude a été conduite dans les villages de Tiéneguebougou (-8.077450 longitude Ouest et 13.573639 latitude Nord) et Farako (8.006489 longitude Ouest et 13.821139 latitude Nord), Guémou (-9.312490 longitude Ouest et 14.546290 latitude Nord) et Bignekolobougou

(-9.375339 longitude Ouest et 14.531990 latitude Nord) au Mali. Les villages de Tiéneguebougou et Farako sont situés dans le cercle de Kolokani, Guémou et Bignekolobougou sont localisés dans le cercle de Diéma (Figure 1).

\section{Caractérisation physico-chimique des sols et cartographie des parcelles d'expérimentation}

Des échantillons composites de sol ont été prélevés dans les parcelles d'essai. Quatorze (14) échantillons ont été prélevés dans chaque parcelle à $0-10 \mathrm{~cm}$ et $10-20 \mathrm{~cm}$ 
de profondeur suivant un dispositif en Astérix (sept points de prélèvement) soit au total deux cent quatre-vingt (280) échantillons pour l'ensemble des 20 parcelles expérimentales). Les échantillons de sol ainsi collectés ont été homogénéisés pour enfin ne retenir qu'un échantillon composite par horizon soit un total de 40 échantillons. Le tout envoyé au laboratoire Sol - Eau- Plante de Sotuba au Mali pour analyses physico-chimiques. Ces analyses qui ont porté sur l'azote, le phosphore, le potassium, le carbone, le $\mathrm{pH}$ (eau), la capacité d'échange cationique (CEC) et les bases échangeables $(\mathrm{Ca}, \mathrm{Mg}, \mathrm{Na})$ ont permis de rendre compte de la situation de référence de la fertilité des sols.

Le pHeau a été dosé par la méthode potentiométrique ; la matière organique $(\% \mathrm{C})$ par la méthode Anne modifiée; l'azote total $(\% \mathrm{~N})$ par la méthode de colorimétrie; le phosphore assimilable (ppm) par la méthode Bray II ; la capacité d'échange cationique (meq/100g) par la méthode METSON à partir d'un dosage à l'Acétate d'ammonium.

Le principe de la cartographie des parcelles d'expérimentation a consisté à matérialiser les 4 sommets de chaque parcelle à l'aide d'un ruban gradué suivi d'un géoréférencement du pourtour et des arbres à l'intérieur à l'aide du GPS (Timble Navigation; USA) et d'une matérialisation à l'encre indélébile. Ces informations permettent de renseigner sur la distribution des parcelles expérimentales et des arbres dans le paysage agraire.

\section{Installation des essais Matériel utilisé}

Le matériel végétal était constitué par les semences du sorgho local. Les fertilisants étaient composés de la fumure organique, d'urée $46 \% \mathrm{~N}\left(\mathrm{CO}\left(\mathrm{NH}_{2}\right)_{2}\right)$ et du complexe céréale (17-17-17+4S). L'apron star a été utilisé pour traiter les semences contre la fonte de semis et autres nuisibles. Le matériel technique était composé du niveau automatique pour le relevé topographique des courbes de niveau, du GPS pour la délimitation et la cartographie des parcelles, des pluviomètres pour évaluer la quantité de pluie, du ruban et de la corde pour la délimitation des parcelles, de la balance pour les pesées, de l'appareil photo-numérique pour fixer les évènements.

\section{Aménagement en courbe de niveau (ACN)}

$\mathrm{La}$ technologie d'aménagement en courbe de niveau s'applique à l'échelle du champ du paysan et respecte les droits fonciers. Le matériel technique utilisé était composé de niveau automatique et de piquets pour piqueter les courbes de niveau et la charrue à traction bovine pour la réalisation des billons de semis et des ados suivant les courbes de niveau. $\mathrm{La}$ réalisation de l'aménagement en courbe de niveau comprenait quatre étapes (Traoré, 2012): le diagnostic de situation, le piquetage des courbes de niveau, la réalisation des ouvrages et l'entretien.

Les parcelles ont été divisées en deux parties dont une a été aménagée en courbe de niveau et l'autre non aménagée mais traitées de la même manière pour les travaux de fertilisation et d'entretien cultural (semis, sarclage, buttage).

\section{Diagnostic de situation des parcelles expérimentales}

Une visite dans les champs, avec les paysans, a permis de repérer les voies de circulation de l'eau et les problèmes d'érosion ou d'excès d'eau qui se posent, la pente des champs, la nature du sol des champs (sableux, argileux, gravillonnaire, etc.). Ensuite, un entretien a été fait avec les paysans sur le niveau d'équipement (charrue à bœufs, houe, pelle), la main-d'œuvre disponible, la période d'exécution des travaux, la disponibilité des matériaux (piquets, fumier, semence de sorgho, etc.).

\section{Piquetage des courbes de niveau et des ouvrages}

Le travail de piquetage a été réalisé de manière participative avec les paysans collaborateurs (Figure 2). Le levé de chaque courbe de niveau a commencé à partir du point le plus élevé du champ à traiter. $\mathrm{La}$ distance entre les piquets était de $10 \mathrm{~m}$ maximum, et elle était réduite si la forme de la courbe de niveau était complexe.

\section{Réalisation des ouvrages}

Les ados ont été confectionnés avec une charrue à bœufs (Figure 3). Les ados ont été fabriqués en 3 aller- retours (soit $1 \mathrm{~m}$ de largeur) d'une charrue à bœufs le long des 
piquets. Une zone de protection de $15 \mathrm{~m}$ sépare le bord du champ et l'ados.

\section{Entretien}

Dans presque tous les cas, il se produit des cassures dans les ouvrages, au moins la première année quand la terre fraîchement remuée est meuble. Les réparations ont été faites au fur et à mesure que les pluies fortes causaient des cassures dans les ouvrages. Par contre, la parcelle non aménagée (témoin) a été travaillée selon les pratiques paysannes c'est-à-dire les façons culturales ne suivaient pas la courbe de niveau. Les travaux de fertilisation et d'entretien cultural (semis, sarclage, buttage) ont été effectués de la même manière dans les parcelles aménagées et dans les témoins.

\section{Dispositif expérimental}

Les essais ont été conduits en 2017 dans les villages de Tiéneguebougou, Farako, Guémou et Bignekolobougou dans les champs de 20 paysans correspondant à 20 répétitions. Les traitements étaient les suivants :

T1: pas d'apport de fertilisants

T2: Micro-dose de fumier $(100 \mathrm{~g} /$ poquet de fumier soit $320 \mathrm{~kg} / \mathrm{ha}$ )

T3: Micro-dose d'engrais minéral (6g/poquet de NPK soit 19,2 $\mathrm{kg} / \mathrm{ha}+1 \mathrm{~g} /$ poquet d'urée soit $3,2 \mathrm{~kg} / \mathrm{ha}$ )

T4: Micro-dose fumier + micro-dose engrais minéral $(100 \mathrm{~g} /$ poquet de fumier soit $320 \mathrm{~kg} / \mathrm{ha}$ $+6 \mathrm{~g} /$ poquet de NPK soit 19,2 kg/ha +1 $\mathrm{g} /$ poquet d'urée soit $3,2 \mathrm{~kg} / \mathrm{ha}$ )

Le dispositif expérimental était le split plot avec 20 répétitions. Les parcelles principales étaient constituées par les parcelles aménagées en courbe de niveau et celles non aménagées (témoin). Les parcelles secondaires étaient formées par 4 niveaux de fertilisation mentionnés dans les traitements. Les parcelles ont été divisées en 2 parties dont une partie a été aménagée en courbe de niveau et l'autre partie n'a pas été aménagée. Les 4 traitements ont été appliqués de la même manière dans les parcelles aménagées et dans les témoins. Les parcelles ont été labourées en billons en suivant les courbes de niveau pour les parcelles aménagées et suivant le sens de la pente du terrain pour les parcelles non aménagées. Les parcelles élémentaires ont été délimitées. La dimension d'une parcelle élémentaire était de $150 \mathrm{~m}^{2}(30 \mathrm{~m} \times 5 \mathrm{~m})$. La distance inter-parcellaire était de $1 \mathrm{~m}$. Celle des inter-blocs était de $2 \mathrm{~m}$. La fumure organique $\left(100 \mathrm{~g}\right.$ poquet ${ }^{-1}$ soit $\left.320 \mathrm{~kg} \mathrm{ha}^{-1}\right)$ et le NPK 17-17-17 (6g poquet ${ }^{-1}$ soit $19,2 \mathrm{~kg} \mathrm{ha}^{-}$ $\left.{ }^{1}\right)$ ont été apportés en micro-dose au moment du semis. Les semences ont été prétraitées à l'apron star (20\% Thiamethoxam, 20\% Metalaxyl-M et 3\% Difénoconazole) à la dose de $250 \mathrm{~g} / 100 \mathrm{~kg}$ de semences. Le semis a été réalisé en juillet 2017 avec un écartement de $80 \mathrm{~cm}$ entre les lignes et $40 \mathrm{~cm}$ entre les poquets. L'urée $(46 \% \mathrm{~N})$ a été enfouie en micro-dose $\left(1 \mathrm{~g}\right.$ poquet $^{-1}$ soit une pincée à trois doigts soit l'équivalent de $3,2 \mathrm{~kg} \mathrm{ha}^{-1}$ ) à $10 \mathrm{~cm}$ des plants de sorgho en début de montaison après une pluie assurant l'humidité du sol. Vingt pluviomètres ont été installés dans les parcelles expérimentales dont un pluviomètre par parcelle pour quantifier la pluviométrie (Figure 4).

\section{Récolte}

La récolte a eu lieu en novembre 2017 après l'arrêt total des pluies. Elle a été réalisée traitement par traitement dans les parcelles aménagées et celles du témoin en éliminant les lignes de bordures. La parcelle utile était de $32,5 \mathrm{~m}^{2}$. Les paramètres étudiés ont porté sur le nombre de panicules pleines à la récolte, le poids des panicules, le poids de la paille, le poids de 1000 graines et le poids des grains. La balance romaine a été utilisée pour les pesées de la paille et des panicules. La balance électronique a été utilisée pour évaluer le poids des grains et de celui de 1000 graines. L'évaluation du poids de 1000 graines était précédé du comptage de 1000 graines à l'aide d'un appareil appelé compteur graines. Si le poids de 1000 graines est élevé, cela signifie un bon remplissage des graines et par conséquent un rendement élevé.

\section{Analyses statistiques}

Les données collectées ont porté sur le nombre et le poids des panicules, le poids de 1000 graines, le poids grains et de la paille. Les données ont été saisies dans Excel puis analysées à l'aide du logiciel d'analyse statistique GenStat version 12.1 en utilisant la méthode d'analyse de variance et le classement des moyennes avec le test de la ppds au seuil de $5 \%$. 


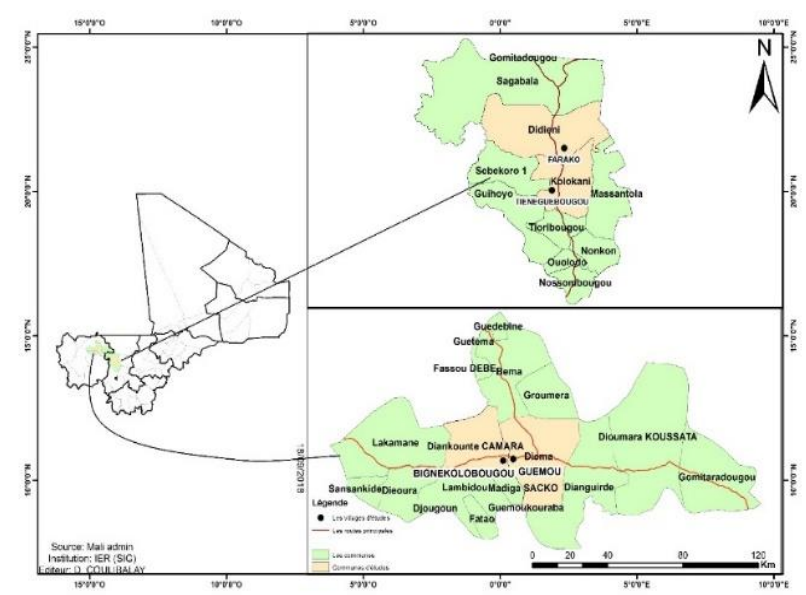

Figure 1: Localisation des sites d'étude.
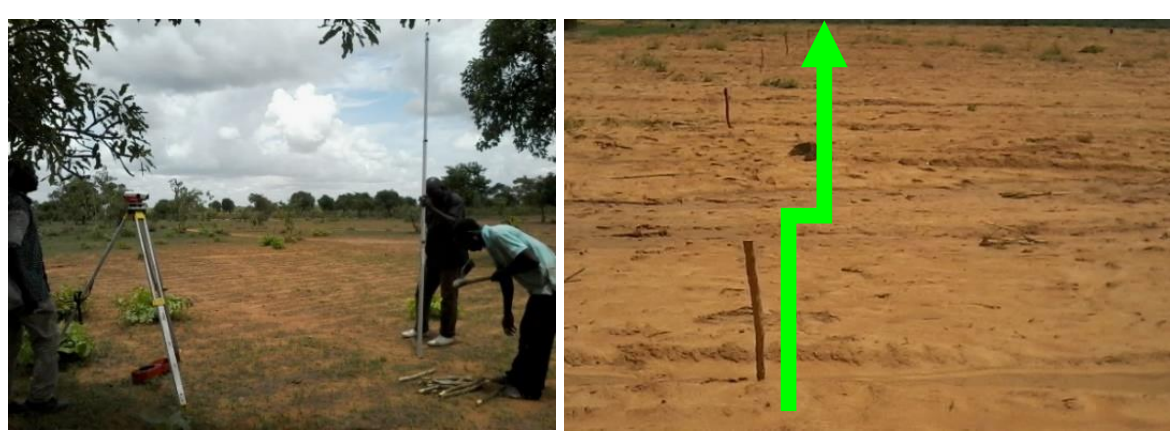

Figure 2: Piquetage des courbes de niveau en 2017.

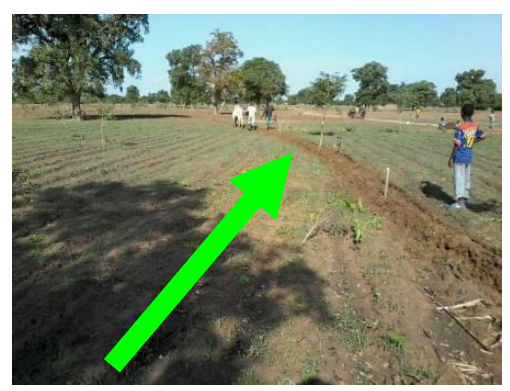

Figure 3 : Confection de l'ados de niveau en 2017 à Tiéneguebougou (Kolokani).

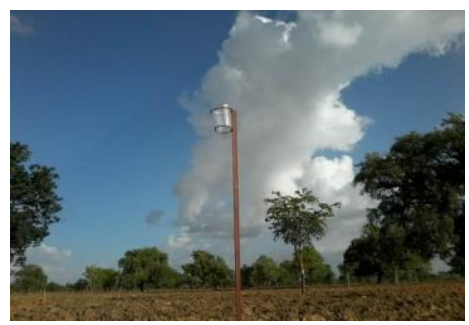

Photo 4: Pluviomètre installé dans la parcelle de Naba Diarra à Tiénéguebougou en 2017. 


\section{RESULTATS}

\section{Caractérisation physico-chimique et cartographie des parcelles expérimentales}

D'un point de vue global, l'ensemble des sites d'étude de Tiéneguebougou, Farako, Guémou et Bignekolobougou (Tableaux 1 et 2) présentent des contraintes physicochimiques quasi-analogues. Il s'agit des sols à texture sableuse à limono-sableuse. Les sols sont faiblement acides avec des valeurs de $\mathrm{pH}$ eau variant de 5,59 à 5,68 (Tableau 1). Le taux de matière organique est faible dans les sols avec des valeurs comprises entre 0,42 et 0,45 . Les sols sont pauvres en azote et en phosphore où les taux varient de 0,03 à 0,01 $\%$ et de 2,18 à 2,19 ppm respectivement (tableau 2). La réserve en éléments nutritifs exprimée par la capacité d'échange cationique (CEC) est moyenne dans les sols où les teneurs en calcium, magnésium et potassium sont très faibles. Le taux de sodium échangeable est très faible (Tableaux 1 et 2).

Les Figures 5 et 6 montrent les points de prélèvement des échantillons de sol et la distribution des ligneux dans les parcelles d'expérimentation.
Effets de la technologie d'aménagement en courbe de niveau sur les rendements du sorgho

L'aménagement en courbe de niveau a permis une augmentation significative ( $\mathrm{p}<$ $0,001)$ des rendements grain et paille, du nombre des panicules et du poids des panicules par rapport à la parcelle témoin (Tableau 3). Les résultats obtenus dans la parcelle aménagée étaient de $845 \mathrm{~kg} \mathrm{ha}^{-1}$ de rendement grain, $2084 \mathrm{~kg} \mathrm{ha}^{-1}$ de rendement paille, 39626 de nombre de panicules ha ${ }^{-1}$ et $1478 \mathrm{~kg}$ de poids panicules $\mathrm{ha}^{-1}$. Ceux de la parcelle témoin étaient de $227 \mathrm{~kg} \mathrm{ha}^{-1}$ rendement grain, $499 \mathrm{~kg} \mathrm{ha}^{-1}$ de paille, 14049 panicules $\mathrm{ha}^{-1}$ et $528 \mathrm{~kg}$ de poids panicules $\mathrm{ha}^{-1}$.

Les rendements panicules, grain, paille et poids de 1000 graines avec l'application de différentes doses de fertilisation sont statistiquement $(\mathrm{p}<0,001)$ différents (Tableau 4). L'apport du fumier et de l'engrais minéral en micro-dose améliore considérablement la production de grain du sorgho comparativement au témoin. Cette fertilisation organo-minérale a produit $1053,6 \mathrm{~kg} \mathrm{ha}^{-1} \mathrm{de}$ rendement grain, $2872 \mathrm{~kg} \mathrm{ha}^{-1}$ de paille, 51763 de panicules ha ${ }^{-1}$ et $1848 \mathrm{~kg}$ de poids panicules.

Tableau 1: Caractérisation physico-chimique des sols de Farako (Kolokani).

\begin{tabular}{|c|c|c|c|}
\hline \multirow[t]{2}{*}{ Paysans } & \multirow[t]{2}{*}{ Analyse réalisée } & \multicolumn{2}{|c|}{ Profondeur } \\
\hline & & $0-10 \mathrm{~cm}$ & $10-20 \mathrm{~cm}$ \\
\hline \multirow[t]{13}{*}{ Gagni Traoré } & $\mathrm{pH}$ & 5.59 & 5.68 \\
\hline & M.O & 0.42 & 0.45 \\
\hline & $\mathrm{N}$ & 0.01 & 0.04 \\
\hline & $\mathrm{P}$ & 1.76 & 1.37 \\
\hline & CEC & 2.47 & 2.21 \\
\hline & $\mathrm{Ca}$ & 0.57 & 0.29 \\
\hline & $\mathrm{Mg}$ & 0.28 & 0.34 \\
\hline & $\mathrm{K}$ & 0.12 & 0.08 \\
\hline & $\mathrm{Na}$ & - & - \\
\hline & Sable & 90 & 90 \\
\hline & Limon & 8 & 8 \\
\hline & Argile & 2 & 2 \\
\hline & C.T & $S$ & S \\
\hline \multirow[t]{2}{*}{ Tiowary Coulibaly } & $\mathrm{pH}$ & 5.23 & 5.01 \\
\hline & M.O & 0.33 & - \\
\hline
\end{tabular}




$\begin{array}{lll}\mathrm{N} & 0.01 & - \\ \mathrm{P} & 2.82 & 2.71 \\ \mathrm{CEC} & 2.33 & 2.74 \\ \mathrm{Ca} & 0.30 & 0.51 \\ \mathrm{Mg} & 0.42 & 0.57 \\ \mathrm{~K} & 0.10 & 0.15 \\ \mathrm{Na} & 0.01 & 0.01 \\ \text { Sable } & 77 & 71 \\ \text { Limon } & 21 & 27 \\ \text { Argile } & 2 & 2 \\ \text { C.T } & \text { LS } & \text { LS }\end{array}$

Légende : $\mathrm{M} . \mathrm{O}=$ matière organique $; \mathrm{C} . \mathrm{T}=$ classe texturale $; \mathrm{CEC}=$ capacité d'échange cationique $;=\mathrm{LS}=$ Limono-Sableux ; $\mathrm{S}=$ Sableux

Tableau 2 : Caractérisation physico-chimique des sols de Guémou (Diéma).

\begin{tabular}{|c|c|c|c|}
\hline \multirow[t]{2}{*}{ Paysans } & \multirow[t]{2}{*}{ Analyse réalisée } & \multicolumn{2}{|c|}{ Profondeur } \\
\hline & & $0-10 \mathrm{~cm}$ & $10-20 \mathrm{~cm}$ \\
\hline \multirow[t]{13}{*}{ Tiéman Coulibaly } & $\mathrm{pH}$ & 5.10 & 5.26 \\
\hline & M.O & 0.18 & - \\
\hline & $\mathrm{N}$ & 0.03 & 0.01 \\
\hline & $\mathrm{P}$ & 2.18 & 2.19 \\
\hline & CEC & 2.24 & 2.32 \\
\hline & $\mathrm{Ca}$ & 0.41 & 0.46 \\
\hline & $\mathrm{Mg}$ & 0.10 & 0.15 \\
\hline & $\mathrm{K}$ & 0.21 & 0.19 \\
\hline & $\mathrm{Na}$ & 0.02 & 0.02 \\
\hline & Sable & 72 & 74 \\
\hline & Limon & 28 & 22 \\
\hline & Argile & 0 & 2 \\
\hline & C.T & LS & LS \\
\hline \multirow[t]{13}{*}{ Mody Coulibaly } & $\mathrm{pH}$ & 5.57 & 5.49 \\
\hline & M.O & 0.23 & 0.16 \\
\hline & $\mathrm{N}$ & 0.01 & 0.02 \\
\hline & $\mathrm{P}$ & 2.52 & 2.52 \\
\hline & CEC & 2.69 & 2.33 \\
\hline & $\mathrm{Ca}$ & 0.68 & 0.35 \\
\hline & $\mathrm{Mg}$ & 0.44 & 0.39 \\
\hline & $\mathrm{K}$ & 0.07 & 0.08 \\
\hline & $\mathrm{Na}$ & 0.00 & 0.01 \\
\hline & Sable & 92 & 92 \\
\hline & Limon & 4 & 6 \\
\hline & Argile & 4 & 2 \\
\hline & C.T & S & $S$ \\
\hline
\end{tabular}

Légende : M.O = matière organique $; \mathrm{C} . \mathrm{T}=$ classe texturale $; \mathrm{CEC}=$ capacité d'échange cationique ; =LS=Limono-Sableux ; $\mathrm{S}=$ Sableux 


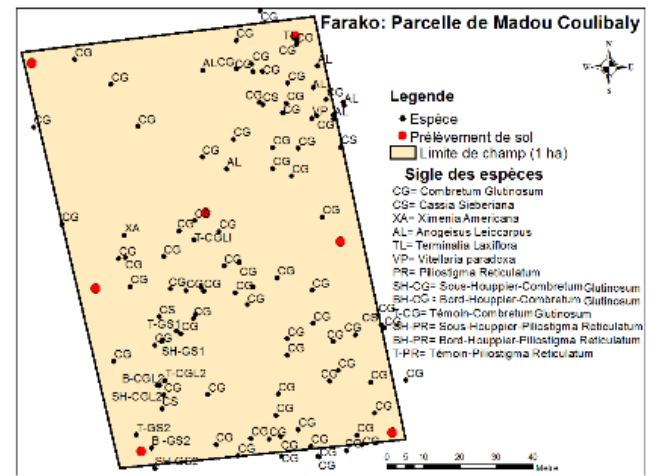

Complément légende $:$ T-GS = témoin Guiera senegalensis, BH-GS = Bord houppier Guiera senegalensis, SH-GS = Sous houppier Guiera senegalensis

Figure 5 : Carte de la parcelle d'essai de Madou Coulibaly à Farako (Kolokani).

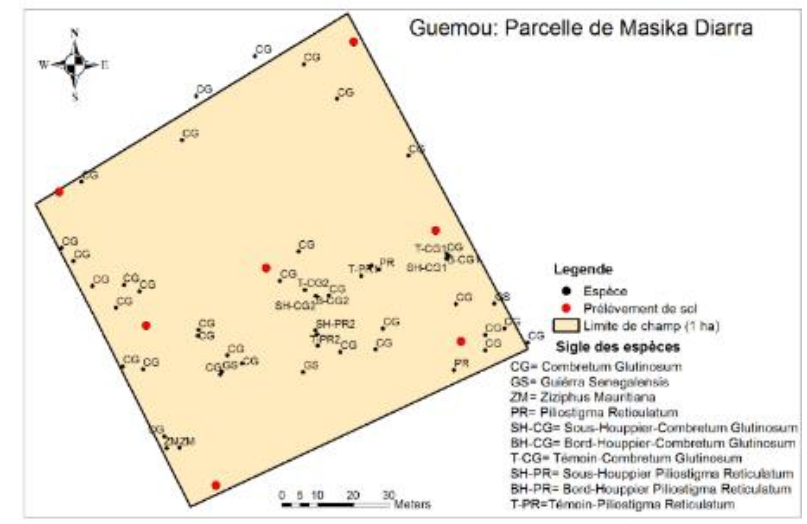

Figure 6 : Carte de la parcelle expérimentale de Massiga Diarra à Bignekolobougou (Diéma).

Tableau 3: Effet de la technologie de l'aménagement en courbe de niveau sur les rendements du sorgho.

\begin{tabular}{|c|c|c|c|c|c|}
\hline Technologies & $\begin{array}{l}\text { Rendement } \\
\text { grain (kg/ha) }\end{array}$ & $\begin{array}{l}\text { Rendement } \\
\text { paille (kg/ha) }\end{array}$ & $\begin{array}{l}\text { Nombre de } \\
\text { panicules }\end{array}$ & $\begin{array}{l}\text { Rendement } \\
\text { panicules } \\
(\mathbf{k g} / \mathrm{ha})\end{array}$ & $\begin{array}{l}\text { Poids de } \\
1000 \text { (g) }\end{array}$ \\
\hline \multicolumn{6}{|l|}{ Situation } \\
\hline $\mathrm{ACN}$ & $845 \mathrm{a}$ & 2084 a & $39626 a$ & $1478 \mathrm{a}$ & $24,61 \mathrm{a}$ \\
\hline NA & $227 \mathrm{~b}$ & $499 \mathrm{~b}$ & $14049 \mathrm{~b}$ & $528 \mathrm{~b}$ & $19,66 \mathrm{a}$ \\
\hline Probabilité & $<0,001$ & $<0,001$ & $<0,001$ & $<0,001$ & 0,884 \\
\hline Signification & HS & HS & HS & HS & NS \\
\hline Ecart-type & 28,64 & 36 & 164 & 32 & 4,71 \\
\hline
\end{tabular}


Tableau 4 : Effets de la technologie d'aménagement en courbe de niveau avec différentes doses de fertilisation sur les rendements du sorgho.

\begin{tabular}{lccccc}
\hline Technologies & $\begin{array}{l}\text { Rendement } \\
\text { grain (kg/ha) }\end{array}$ & $\begin{array}{l}\text { Rendement } \\
\text { paille (kg/ha) }\end{array}$ & $\begin{array}{l}\text { Nombre de } \\
\text { panicules } \\
\text { (nombre/ha) }\end{array}$ & $\begin{array}{l}\text { Rendement } \\
\text { panicules } \\
\text { (kg/ha) }\end{array}$ & $\begin{array}{l}\text { Poids de } \\
\mathbf{1 0 0 0}(\mathbf{g})\end{array}$ \\
\hline Fertilisation & 628,5 c & $1364 \mathrm{~d}$ & $27284 \mathrm{~b}$ & $1077 \mathrm{~b}$ & $22,5 \mathrm{~b}$ \\
T1ACN & $807,2 \mathrm{~b}$ & $1785 \mathrm{c}$ & $35216 \mathrm{~b}$ & $1322 \mathrm{~b}$ & $23,56 \mathrm{~b}$ \\
T2ACN & $890,7 \mathrm{~b}$ & $2314 \mathrm{~b}$ & $44242 \mathrm{a}$ & $1666 \mathrm{a}$ & $25,28 \mathrm{a}$ \\
T3ACN & $1053,6 \mathrm{a}$ & $2872 \mathrm{a}$ & $51763 \mathrm{a}$ & $1848 \mathrm{a}$ & $27,11 \mathrm{a}$ \\
T4ACN & $131,5 \mathrm{~d}$ & $174 \mathrm{f}$ & $8067 \mathrm{~d}$ & $355 \mathrm{c}$ & $18 \mathrm{c}$ \\
T1NA & $199,4 \mathrm{~d}$ & $371 \mathrm{f}$ & $12021 \mathrm{~d}$ & $428 \mathrm{c}$ & $19,22 \mathrm{c}$ \\
T2NA & $266,7 \mathrm{~d}$ & $596 \mathrm{e}$ & $16089 \mathrm{c}$ & $601 \mathrm{c}$ & $20,33 \mathrm{c}$ \\
T3NA & $310,6 \mathrm{~d}$ & $853 \mathrm{e}$ & $20017 \mathrm{c}$ & $726 \mathrm{c}$ & $21,11 \mathrm{~b}$ \\
T4NA & $<0,001$ & $<0,001$ & $<0,001$ & $<0,001$ & $<0,001$ \\
Probabilité & $\mathrm{HS}$ & $\mathrm{HS}$ & $\mathrm{HS}$ & $\mathrm{HS}$ & $\mathrm{HS}$ \\
Signification & $0,88 \mathrm{a}$ & $0,95 \mathrm{a}$ & $0,81 \mathrm{a}$ & $0,99 \mathrm{a}$ & $0,4 \mathrm{a}$ \\
Interaction & & & & & \\
aménagement $*$ & 23,15 & 35,93 & 164 & 32 & 4,71 \\
fertilisation & & 40 & 35,2 & 36 & 10,8 \\
Signification & $\mathrm{NS}$ & $\mathrm{NS}$ & $\mathrm{NS}$ & $\mathrm{NS}$ & $\mathrm{NS}$ \\
Moyenne générale & 536,03 & 1291,12 & 26837,37 & 1003 & 22,14 \\
Ecart-type & 25 & & & & \\
CV\% & & & & & \\
\hline
\end{tabular}

Légende : T1, T2, T3 et T4 = traitements, $\mathrm{ACN}=$ aménagement en courbe de niveau, $\mathrm{NA}=$ non aménagé, $\mathrm{NS}=$ non significatif, $\mathrm{HS}=$ hautement significatif, $\mathrm{CV} \%=$ coefficient de variation.

\section{DISCUSSION}

\section{Caractérisation physico-chimique}

L'analyse des échantillons de sols des sites de Kolokani et de Diéma a montré que les essais ont été implantés sur des sols ferrugineux tropicaux ayant une faible fertilité, une acidité, une carence en phosphore, en azote et en potassium. La caractérisation physico-chimique avait pour but de connaître la fertilité initiale des parcelles d'expérimentation. Des résultats analogues ont été obtenus et rapportés par Traoré et al. (2017b) dans les conditions similaires au Mali.
Effet de la technologie d'aménagement en courbes de niveau sur les rendements du sorgho

L'aménagement en courbe de niveau a permis une augmentation significative $(\mathrm{p}<$ 0,001) du rendement grain, paille, nombre panicules, poids panicules et poids de 1000 graines.

La pluviométrie a été faible en 2017 (369 mm à Kolokani et $240 \mathrm{~mm}$ à Diéma), cependant, l'aménagement en courbe de niveau a augmenté de plus de $175 \%$ les rendements grain, paille, nombre des panicules, poids des panicules et de plus $25 \%$ le poids de 1000 graines par rapport au témoin 
(Tableau 3). Les rendements panicules, grain, paille et poids de 1000 graines des différentes doses de fertilisation étaient statistiquement différents $(\mathrm{p}<0,001)$. La fertilisation organominérale (apport de fumier, urée et NPK) en micro-dose a amélioré considérablement la production de grain $\mathrm{du}$ sorgho comparativement au témoin et était en moyenne de 39\% supérieur aux traitements qui ont apporté du fumier seul ou de l'engrais minéral (Tableau 4). La même formule de fertilisation organo-minérale a augmenté significativement le nombre de panicules par rapport au témoin et a été en moyenne de 53 $\%$ supérieur aux traitements ayant apporté du fumier seul ou de l'engrais minéral seul (Tableau 4). L'apport du fumier et de l'engrais minéral en micro-dose a augmenté de manière significative le rendement paille du sorgho par rapport au témoin et a été en moyenne de 65\% supérieur aux traitements qui ont apporté du fumier ou de l'engrais minéral (Tableau 4). L'apport du fumier et de l'engrais minéral en micro-dose a également eu une influence assez importante sur le poids de 1000 graines comparativement au témoin et était en moyenne de 14\% supérieur aux traitements ayant nécessité l'apport du fumier ou de l'engrais minéral seul (Tableau 4). Les résultats de la réponse à l'aménagement et à la fertilisation organo-minérale dans cette étude sont conformes à des travaux similaires réalisés au Mali par Gigou et al. (2006) ; Traoré et al. (2017a, 2017b, 2003 et 2002), Doumbia et al. (2009). Ces auteurs ont obtenu des améliorations de rendements entre 22 et $60 \%$. Cette augmentation de rendement est due à la plus grande disponibilité de l'eau dans les parcelles aménagées que dans les parcelles témoins. Cette disponibilité en eau dans les parcelles serait due à l'effet de l'aménagement qui favorise la solubilisation des fertilisants et leur mise à la disposition des cultures (Traoré et al., 2017b ; Birhanu et al., 2018). Selon Traoré et al. (2017b et 2017a), l'aménagement en courbe de niveau réduit le ruissellement et améliore l'alimentation hydrique des cultures. Il en résulte un effet net sur les rendements en années sèches. En effet, la présente étude a été réalisée en année sèche en 2017 (369 mm à Kolokani et $240 \mathrm{~mm}$ à Diéma), néanmoins les rendements grain et paille ont été augmentés de plus de $175 \%$ dans les parcelles aménagées par rapport aux témoins.

\section{Conclusion}

Les objectifs de cette étude consistaient à caractériser les propriétés physicochimiques des sols et à évaluer l'effet de l'aménagement en courbe de niveau sur les rendements du sorgho. L'ACN a produit 845 $\mathrm{kg} \mathrm{ha}^{-1}$ de rendement grain et $2084 \mathrm{~kg} \mathrm{ha}^{-1} \mathrm{de}$ paille contre $227 \mathrm{~kg} \mathrm{ha}^{-1}$ et $499 \mathrm{~kg} \mathrm{ha}^{-1}$ pour le témoin. L'ACN a augmenté de plus de $175 \%$ les rendements grain et paille par rapport au témoin. La fertilisation organo-minérale en micro-dose a augmenté de $39 \%$ et $53 \%$ les rendements grain et paille comparativement au témoin. La technologie d'ACN permet de doubler voire tripler les rendements grain et paille des cultures par rapport au témoin. Cette technologie est reproductible partout au Sahel où la pluviométrie est faible et dans d'autres zones à écologie similaire.

\section{CONFLIT D'INTERETS}

Les auteurs de l'article déclarent qu'il n'y a pas de conflit d'intérêts pour ce travail.

\section{CONTRIBUTIONS DES AUTEURS}

MZ a assuré la conduite des différentes activités sur le terrain et a rédigé l'article ; KT a encadré la mise en œuvre des activités. Il a lu et amélioré l'article; MF a supervisé les travaux de recherche et contribué à l'amélioration de l'article ; BSM a participé aux travaux sur le terrain comme appui à la recherche; OS a participé à la réalisation des activités sur le terrain; MAT a facilité le déblocage des fonds destinés au financement des travaux de recherche. Il a suivi le déroulement des activités.

\section{REMERCIEMENTS}

Nous remercions vivement tous ceux qui nous ont apporté leur appui scientifique dans la réalisation des travaux de recherche et la production $\mathrm{du}$ présent article. Qu'ils 
trouvent ici l'expression de notre très haute reconnaissance.

\section{REFERENCES}

Birhanu ZB, Kalifa T, Murali KG, Félix B, Ramadjita T, Anthony MW, 2018. A watershed approach to managing rainfed agriculture in the semiarid region of southern Mali: integrated research on water and land use. Environ Dev Sustain. https://doi.org/10.1007/s10668-0180144-9

Gigou J, Traore K, Giraudy F, Coulibaly H, Sogoba B, Doumbia M. 2006. Aménagement paysan des terres et réduction du ruissellement dans les savanes africaines. Cah Agric, 15(1): 116-122.

Kablan R, Yost RS, Brannan K, Doumbia M, Traore K, Yorote A, Toloba Y, Sissoko S, Samake O, Vaksman M, Dioni L, Sissoko M. 2008. Aménagement en courbes de niveau, increasing rainfall capture, storage, and drainage in soils of Mali. Arid Land Res Manag, 22: 62 - 80.

Kizito F, Sène M, Dragila MI, Lufafa A, Diedhiou EDI, Cuenca R, Selker J, Dick RP. 2005. Seasonal soil water variation and root dynamics between two semiarid shrubs coexisting with Pearl millet in Senegal. West Africa. J. Arid Environ., 67: 436 - 455.

Doumbia M, Jarju A, Sène M, Traoré K, Yost R, Kablan R, Brannan K, Abou B,
Yamoah C, Querido A, Traoré PCS, Ballo A. 2009. Sequestration of organic carbon in West African soils by Aménagement en Courbes de Niveau. Agronomy for Sustainable Development, 29(2): 267-275.

Traoré K, Sidibé DK, Coulibaly H, Bayala J. 2017b. Optimizing yield of improved varieties of millet and sorghum under highly variable rainfall conditions using contour ridges in Cinzana, Mali. Agriculture and Food Security, 6: 11. DOI 10.1186/s40066-016-0086-0.

Traoré K, Sidibe DK, Coulibaly H. 2017a. Climate Smart Agriculture as Final Goal: Use of Improved Cereals Varieties in Cinzana, Mali. Journal of Agricultural Studies, 5(1). DOI:10.5296/jas.v5i1.

Traoré KB, Gigou J, Yamada M, Samaké O, Coulibaly H, Doumbia M. 2012. Aménagement en courbes de niveau pour la conservation des sols en champs paysans. $16 \mathrm{p}$.

Traoré K. 2003. Le parc à karité, sa contribution à la durabilité de l'agrosystème : cas d'une topo séquence à Konobougou dans le Mali-sud. Thèse de Doctorat en science du sol. Université de Montpellier II, ENSAM, 180 p.

Traoré K. 2003. Le parc à karité (Vitellaria paradoxa - Gaertner f.) et fertilité des sols de la zone Mali-sud. DEA National de Sciences du Sol, Université Henry Poincaré, Nancy: 20 p. 